\title{
Das Alte Testament im Neuen.
}

\section{Von Theodor Haering, Tübingen. ${ }^{1}$}

Vor 32 Jahren erschien W. Herrmanns Schrift „Warum bedarf unser Glaube geschichtlicher Tatsachen?" Die Älteren unter uns erinnern sich noch lebhaft des ersten Eindrucks: „klein nach der Zahl der Worte, groß nach dem Gewicht der Gedanken“. Die Schrift war das Seitenstück zu der 10 Jahre früher erschienenen "Die Metaphysik in der Theologie". Beide zusammen bezeichnen programmatisch die beiden Pole Herrmannscher Theologie; die Ausführung des letzteren Grundgedankens brachte „Die Religion im Verhältnis zum Welterkennen und zur Sittlichkeit“, die des ersten „Der Verkehr des Christen mit Gott" wenn man nur eben auf die Hauptsache sieht.

In den Bereich jenes ersten großen Themas gehört der folgende Versuch. Es ist bekannt, wie die Verhandlungen über "Glaube und Geschichte" oft ausdrücklich, noch öfter tatsächlich um jenes Herrmannsche Losungswort sich bewegten, nicht selten auch in persönlicher Liebe und Abneigung, wie es beim Kampf um eine persönlich vertretene Wahrheit verständlich ist. Auf der ganzen Linie hält der Kampf an. Mit neuem Mut, auch mit neuen Kampfmitteln ist die Jugend in diesen Kampf eingetreten, auch sie wird ihn nicht zu Ende führen. Denn, einmal entbrannt, kann er nicht aufhören, bis das eine oder andere Heer einen entscheidenden Sieg erfochten hat. Und zwar. hieße das, für den Fall, daß „unser Glaube geschichtlicher Tatsachen nicht mehr bedarf": er wäre ein anderer Glaube geworden: .Die Frage, klar gestellt, ist seine Schicksalsfrage. Aber nicht mit dieser Tiefe des Problems beschäftigen sich die folgenden Blätter. Nur mit einer Seitenfrage, wenigstens nach dem nächsten Eindruck. Wenn die innere

1 [Mit diesem Beitrag eines Fachgenossen des Jubilars darf sich auch die Zeitschr. in die Reihe derer stellen, die Wilhelm Herrmann (geb. 6. Dez. 1846) zu seinem 70. Geburtstag herzlich Glück wünschen. Liegt auch der Schwerpunkt seiner Lebensarbeit auf anderem Gebiet, so darf die neutestamentliche Wissenschaft doch dankbar an diesem Tag der Anregungen gedenken, die auch sie von ihm empfangen hat. E. P.]

Zeitschr. f. d. neutest. Wiss. 17. Jahrg. 1916. 
Unlösbarkeit unsres Glaubens von der Geschichte verhandelt wird, muß doch immer der Blick auch auf die Glaubwürdigkeit der Geschichte sich richten; selbst wenn diese, wie W. Herrmann von Anfang an betont hat, nur dem Glauben feststeht, feststeht in dem Sinn, in welchem sie zum Erlebnis des Glaubens selbst gehört. Aber auch nicht diese Frage in ihrer vielverzweigten Mannigfaltigkeit soll hier aufgerollt, nur ein ganz umgrenzter Beitrag dazu versucht werden; eben durch die Erinnerung an die alttestamentliche Bedingtheit der neutestamentlichen Zeugnisse.

Wenn nur vom Alten und Neuen Testament die Rede ist, so will damit nicht die Ausdehnung der Untersuchung auf die zwischen beiden liegende Literatur, ebensowenig auf die dem NT nächstfolgende irgend abgelehnt werden, entgegen dem Wesen einer geschichtlichen Untersuchung. Im folgenden ist von selbst die Gelegenheit, darauf hinzuweisen; falls zugleich ein wenn auch noch so kleiner Beitrag für die Frage nach Recht oder Unrecht jener Abgrenzung sich ergibt, desto besser. Wichtiger ist eine andere Voraussetzung, die auch erst durch die Erörterung begründet werden kann, und zwar eine doppelseitige. Einmai für den Umfang der alttestamentlichen Bedingtheit des NTs. Noch immer ist nicht vollständig genug das Verfahren aufgegeben, allein oder in erster Linie die sogenannte "messianische Weissagung“ ins Auge zu fassen. Gerade dadurch ist die wirkliche alttestamentliche Bedingtheit des Neuen verdeckt worden; die weithin berechtigte Gegnerschaft gegen jenes Verfahren führte $z u$ der Selbsttäuschung, in der Polemik die ganze Aufgabe gelöst $z u$ haben. Mit der bewußten Erweiterung des Umfangs hängt die veränderte Art der Behandlung zusammen. Sie muß die rein geschichtliche, von keinem vorgefaßten Wunsch beeinflußte sein. Das Ideal der vorgeschlagenen Untersuchung ist dasselbe, welches jeder religionsgeschichtlichen Untersuchung vorschweben muß; sie selbst möchte nichts sein als der Hinweis auf ein Stück religionsgeschichtlicher Vergleichung.

Wir versuchen einige methodische Regeln für den Vollzug dieser Untersuchung $z u$ gewinnen. Sodann soll ihre Bedeutung für die einzelnen theologischen Disziplinen aufgezeigt werden.

Also zuerst zur Methode! Voran darf der Satz stehen: Das Ideal wäre verwirklicht, wenn das ganze Alte und das ganze Neue Testament unter dem angegebenen Gesichtspunkt untersucht wäre. Nur in dieser Doppelbetrachtung erkennen wir den vollständigen Sachverhalt. Etwa wie wir eine Landschaft tiefer erfaßt haben, wenn wir sie im Morgen- 
und im Mittagslicht betrachten konnten, so die alttestamentliche Bedingtheit des NTs, wenn wir sie von den wirksamen Kräften des Alten aus und von den Wirkungen im Neuen aus verstehen. Oder wie wir, wenn wir die Jugend eines großen Menschen in seinem Mannesalter wirksam aufzeigen wollen, vom Jugendbild und vom Mannesbild werden ausgehen müssen. Gingen wir allein vom AT aus, so liefen wir Gefahr, vielleicht wichtige Einwirkungen auf das NT nicht zu beachten, weil keine ausdrücklichen Bezugnahmen offen zutage liegen, etwa in bezug auf den Begriff "Herrlichkeit" oder in bezug auf das "Leiden zugunsten anderer", wenn man bei den Zitaten stehen bleibt. Umgekehrt, gingen wir nur vom NT aus, so würde die wichtige Beobachtung, wie viele Stoffe des ATs im NT mit Stillschweigen übergangen, in ihm nicht wirksam geworden sind, nicht so entscheidend hervortreten, wie es die Sache fordert. Nun ist aber klar, welche Arbeit eine solche Gesamtvergleichung beider Testamente von beiden aus umschlösse. Tatsächlich wird es sich für den einzelnen Arbeiter nur darum handeln können: er faßt die Aufgabe vom AT oder vom NT aus an; und auch das wieder mit einer Beschränkung: er untersucht einzelne Bücher des ATs in ihrer Wirkung, sei es auf das ganze NT, sei es auf einzelne neutestamentliche Schriften, oder umgekehrt einzelne Schriften des NTs in ihrer Bedingtheit durch das AT, bzw. durch einzelne Schriften des ATs. Z. B. ist besonders lehrreich die Wirkung des II. Jesaja oder der Psalmen auf das NT auf das Bewußtsein Jesu, auf die Apokalypse. Ungemein verschieden ist die Wirkungskraft der einzelnen Bücher des ATs auf das NT, quantitativ und qualitativ. Jesaja verhält sich, ganz äußerlich betrachtet, zu Jeremia etwa wie $3: 1$, womit aber keineswegs ganz besonders tiefe Wirkung des letztern ausgeschlossen ist, z. B. etwa Jer 31, 31 im Verhältnis zum Abendmahlswort; Jesaja wirkt im Hebräerbrief so gut wie gar nicht, die Psalmen in der Apokalypse stark, aber nur nach einer bestimmten Seite. Im folgenden gehen wir vom AT aus und suchen seine Wirkungen im NT uns zu verdeutlichen.

Fragen wir genauer nach den einzelnen Gesichtspunkten, unter denen wir die unendlich mannigfaltige alttestamentliche Bedingtheit des NTs einigermaßen ordnen mögen, so seien uns die einfachsten willkommen: Was? Wer? Wie? d. h. welche Stoffe des ATs sind im NT verwertet? Von wem? In welcher Weise? Nur muß von vornherein nachdrücklich betont werden: diese Fragen greifen ineinander über. Bei der Besprechung des „Wer?" kommt immer noch das „Was?" und bei beiden schon das "Wie?" in Betracht, ja beide können erst beim 
„Wie?“ abgeschlossen werden, auch wenn darauf im folgenden nicht immer aufmerksam zụ machen ist.

Das „Was?", die Frage nach dem im NT wirksam sich erweisenden alttestamentlichen Stoff darf, wie schon im voraus betont werden mußte, nicht auf den im engern oder weitern Sinn "messianischen“ Inhalt des ATs beschränkt werden. Dadurch entstünde ein völlig einseitiges Bild des wirklichen Einflusses, der vom AT auf das NT ausgeht; und es ist bekannt, wie oft und vielfältig die Enge dieses Sehwinkels die Erkenntnis des ATs und des NTs gehindert hat. Vielmehr ist die ganze religiöse Gedankenwelt des ATs in Betracht zu ziehen. Jedes zufällig herausgegriffene Beispiel führt dabei zu Überraschungen. In 1 Chron 29, 10ff. findet man einen großen Teil Vaterunserworte oder ihre Äquivalente. In Jesaja 40 ist Trost, Herrlichkeit, Heil, Frohbotschaft, Hirte, Geist und Fleisch, lieben und erwählen nur eine Auswahl von Wurzelworten und -gedanken für das NT. Aber dann allerdings, gerade wenn man nicht auf "messianische" Beziehungen ausgeht, ist der Gesamteindruck nur um so stärker, wie zukunftsmächtig, vorwärtsdrängend unzählige alttestamentliche Worte sind, ja, recht verstanden, alle, gerade etwa die eben erwähnten. Die religionsgeschichtliche Beobachtung, wie eigenartig die Religion Israels auf ihrer prophetischen Stufe Religion der Geschichte ist, wie sie nicht unter dem „ästhetischen“ Vorzeichen des Blühens und Verwelkens in der Natur steht, sondern unter dem „teleologischen“,_diese Beobachtung, der Historiker wie Ranke und Philosophen wie Lotze feinfühligen Ausdruck gegeben haben, wird von einer Untersuchung, wie der unsrigen, im umfassendsten Maß bestätigt. Und dann gewinnt man auch für die im engeren Sinn messianischen Aussagen und ihre Unterschiede das richtige Augenmaß. U. a. wird jene doppelte Linie der Hoffnung wichtig, nach deren einer Israels Gott selbst die Heilszeit heraufführt, nach deren anderer in dem von ihm erwählten, ausgerüsteten, geleiteten menschlichen Vertreter; und bei den auf dieser letzteren Linie liegenden Aussagen ist man dann von selbst vor der Beschränkung auf das alte Schema des vollendeten Königs, Priesters, Propheten bewahrt.

Fruchtbarer wird die Frage, "was“ aus dem AT im NT wirksam geworden, durch unsre zweite: "wer" im NT diese Einwirkung zeigt, welche Schriftengruppe, welche Zeit? Ganz von selbst bietet sich hierbei die Unterscheidung zwischen den in unsern Gemeindezeugnissen auf Jesus selbst zurückgeführten Verwertungen des ATs und denen der Gemeinde selbst. Und gerade wenn wir, der geschichtlichen Wirklichkeit gemäß, nicht sofort Zeugnisse Jesu und der Gemeinde 
unterscheiden, weil wir jene nur in diesen haben, werden wir vielleicht einen wertvollen Beweis für das Recht jener Unterscheidung gewinnen, so wie er einer objektiven Untersuchung zugänglich ist, und dadurch ein Mittel, das Ursprüngliche vom Abgeleiteten genauer zu sondern. Wenn hierbei, wie es sich versteht, die Worte Jesu bei den Synoptikern und im vierten Evangelium besonders auf ihre alttestamentliche Bedingtheit hin geprüft werden, so wird sich z. B. nicht leugnen lassen, daß ausdrückliche Bezugnahme auf "messianische" Stoffe des ATs in den synoptischen Logien häufiger sich findet als bei Johannes, und daß diese Stoffe, was beim „Wie?" der Verwendung bestimmter ins Auge $\mathrm{zu}$ fassen ist, in andrer Art verwendet sind (vgl. Mt 11, 14; 26,$31 ; 27,46$; Lc 22,37), ferner daß die ganze religiöse Gedankenwelt dort unmittelbarer alttestamentlich bedingt ist als hier, man vergleiche "Reich", „Gerechtigkeit" u. a. mit "Licht", „Leben“. (Damit ist gewiß nichts an der sonst oft gemachten Beobachtung abgemindert, daß im Verhältnis z. B. zu den apostolischen Vätern auch das vierte Evangelium starke Wurzeln im AT hat.) Nicht gleichgültig ist ferner die besondere Frage, ob in den Synoptikern selbst gewisse alttestamentliche Beziehungen in den einzelnen Stadien der Geschichte Jesu, sofern man überhaupt von solchen reden kann, stärker hervortreten; man denke an die Untersuchungen, ob "der Menschensohn" nur den Ausgängen zugehöre. Wichtiger als diese Gesichtspunkte ist sodann der dabei schon berührte, aber im einzelnen durchzuführende Nachweis, was von den oben unterschiedenen Stoffen von Jesus angeeignet ist. Eine Statistik über die häufigere oder seltenere Benutzung der einzelnen alttestamentlichen Schriften bildet eine notwendige Vorarbeit. Hierbei ergäbe sich von selbst das unmöglich bloß zufällige Zurücktreten ganzer Gruppen und einzelner sehr bald in der Gemeinde wichtig gewordener Gedanken. Auch daran wird bei dem „Wie?" der Verwendung zu erinnern sein.

Ein derartiges Verfahren wäre dann, auf die Zeugnisse der Gemeinde, im einzelnen abgewandelt, gieichfalls anzuwenden. In betreff der statistischen Ergebnisse kann wenigstens erwähnt werden, wie eigentümlich sie sich gestalten, etwa für Römer- und Hebräerbrief im Vergleich mit den Pastoralbriefen oder im Vergleich untereinander, gerade wenn die zuvor angegebenen Gesichtspunkte sämtlich verwendet werden.

Bei der Frage nach dem „Wie?“ der Verwendung mögen die das Äußerliche betreffenden Gesichtspunkte voranstehen; sie sind zum Teil doch auch nicht ganz ohne sachlichen Wert. So z. B., wenn wir uns 
an das „Was?" und „Wie?" zurückerinnern, die verhältnismäßige Seltenheit der ausdrücklichen Berufung auf das AT seitens Jesu, verglichen mit der oft so gehäuften in den Gemeindezeugnissen; vielleicht darf man auch darauf aufmerksam machen, daß das "es ist geschrieben" mit dem "habt ihr nicht gelesen?" und "wenn ihr es annehmen wollt" wechselt, während das "es sagt einer irgendwo" fehit, vollends nicht, wie im Hebräerbrief, Anreden an Gott als Gottessprüche selbst behandelt werden. Weiterhin ist $\mathrm{zu}$ erinnern an das Verhältnis von Grundtext und griechischer Übersetzung. In Mt 3, 3 ist die Stellung der Worte „in der Wüste" geradezu entscheidend; umgekehrt wird ausnahmsweise auf den hebräischen Text zurückgegriffen, wenn dieser für den Schriftbeweis passender ist, wie Röm 1, 17 (das fehlende „mein“). Bedeutsamer ist die eigentlich hermeneutische Stellung zum AT. Sein allegorischer Gebrauch, bei Paulus Gal 4, 24 ausdrücklich hervorgehoben (freilich noch immer von einzelnen „Erklärern“ dem Paulus abgesprochen) und tatsächlich auch 1 Kor 10, 4; 1 Kor 9,9f.; Gal 3, 16 geübt, im Hebräerbrief aber viel umfassender und nach allen Regeln der Kunst (z.B. argumentum ex silentio in 7,3) angewendet, dann bald, vgl. Barn. 9, $7 \mathrm{ff} .$, maßlos und sinnlos ausgebaut, läßt sich bei Jesus nicht nachweisen, denn Mt 22,32 im Vergleich mit 2 Mos 3,6 ist deutlich eine andersartige, sogleich $\mathrm{zu}$ besprechende Verwendung. Neben dem wörtlichen und neben dem allegorischen Verständnis durchzieht alle Gruppen neutestamentlicher Schriften jene Beziehung der vollendeten Offenbarung auf die vorbereitende in bezug auf Personen, Einrichtungen, Wahrheiten, für die man doch noch immer kein besseres Wort als "typische“ gefunden hat, wenn nur die seltsame Ablehnung der allegorischen Deutung grundsätzlich aufgegeben wird. In den einzelnen Stellen wird man freilich über die Abgrenzung beider Arten naturgemäß immer streiten.

Tiefer als diese formalen Gesichtspunkte führt die innere Art der Verwendung des ATs im NT. Indem der Blick oft ausschließlich auf dem in der Tat wichtigsten Gesichtspunkt des "Schriftbeweises" haften bleibt, übersieht man leicht, daß eine Fülle neutestamentlicher Stellen das AT nur einfach als Kleid des ausgesprochenen religiösen Gedankens benützt, ähnlich wie wir selbst das AT und NT oder weiterhin bekannte Worte unsrer religiösen oder auch weltlichen Klassiker in Prosa und Poesie, Spruchweisheit usw. Man denke an Lc 1, 46ff. vgl. mit 1. Sam 2, 1 ff.; 1 Pt 3, 10ff. vgl. mit Ps 34, 13ff; Röm 8, 36 vgl. mit Ps 44, 23; Röm 8,33 mit Jes 50,8.9. Nur liegt es in der Natur der Sache, daß der Übergang derartiger Verwendung in die des Schriftbeweises oft 
fließend ist. Beim Schriftbeweis selbst aber werden wir, in Erinnerung an unsre Besprechung des „Was?", ausdrücklich unterscheiden müssen den Beweis für religiöse Wahrheiten überhaupt und den Beweis für die Erfüllung alttestamentlicher Weissagungen. Ersteres gilt z.B. von Röm 4, $7 \mathrm{ff}$. im Verhältnis zu 1 Mos 15, 6 oder Mt 22, 32 im Verhältnis zu 2 Mos 3,6, Letzteres von Mt 22, 41 vgl. mit Ps 110,1. Bei Ps 118, 22 vgl. mit Mt 21, 42 mag man zunächst zweifelhaft sein, wohin man die Stelle ziehen soll. Im Hinblick auf diesen entscheidenden Gesichtspunkt des Schriftbeweises wird eine ins einzelne gehende Versenkung in die alttestamentliche Bedingtheit der neutestamentlichen Aussagen vor die stets beobachtete und beachtete Tatsache doch immer mit neuer und vertiefter Verwunderung stellen, wie fest, aber auch wie beweglich jener Zusammenhang ist. Die Bedeutung der "Buchreligion“ überhaupt, dann das besondere Judenerbe der Buchreligion nach der Seite der Gebundenheit und der Freiheit kommt von immer neuen Seiten in immer tieferen Beziehungen zum Bewußtsein. Fast keiner der oft gewagten entgegengesetzten Allgemeinsätze ist als völlig falsch $\mathrm{zu}$ bezeichnen: es sei nichts Neues im NT, es sei alles neu. Aber nun werden die Gründe deutlich, warum überhaupt so Entgegengesetztes behauptet werden konnte. Und das grundsätzlich richtige Urteil Bengels „Novum Testamentum in Vetere latet, Vetus in Novo patet", das dann ohne genaue methodische Untersuchung bei manchen seiner Nachfolger wieder der alten willkürlichen Identifizierung den Weg bereitete, wird durch eine methodisch strenge Untersuchung erst fruchtbar. Nennen wir nur wieder ein Beispiel, und zwar aus dem wichtigsten Gebiet, aber gewissermaßen ein unscheinbares Beispiel. In der Erzählung von der Versuchung Jesu wird das Psalmwort vom "Auf den Händen getragen werden", das in der Geschichte der christlichen Frömmigkeit, auch jetzt im großen Krieg, so bedeutsam geworden ist, durch das andere Schriftwort "Du sollst Gott, deinen Herm, nicht versuchen " beschränkt, in seiner äußerlichen Anwendung auf den vorliegenden Fall außer Geltung gesetzt. Die echte praktische Anwendung hat auf dieses "Schrift wider Schrift" oft nachdrücklich hingewiesen, ohne doch immer auch nur praktisch die richtigen Folgerungen $z \mathfrak{u}$ ziehen, noch weniger ihrer für das Verständnis Jesu sich bewußt $\mathrm{zu}$ werden. Kurz: keines seiner Worte ist ohne Heimat im AT, das AT ist seine Heimat. Aber als der "Sohn" schaltet er frei in diesem Haus des "Vaters" mit vielen Wohnungen, so gewiß er an den Worten des AT vom "Sohn" sich über sein Innerstes, Eigenstes deutlich geworden ist; alles Stoffliche dient zur Nahrung der Flamme, und das nicht Anzueignende wird mit königlicher Selbstverständlichkeit 
stillschweigend beiseite gelegt. Wenn man das Gleichnis nicht miBverstehen will: hier ist die größte Heterogonie der Zwecke, die uns die Geschichte bietet. Auch sonst ist es das Siegel der ganz Großen, daß sie die Vergangenheit nicht meistern wie die eingebildeten Kleinen, nicht vermeinen, als seien sie nur schöpferischer Anfang, daß sie vielmehr, oft sich selbst gerade in ihrem Größten ein Geheimnis, verwundert vor ihrer Tat stehen. Aber alles das ist doch im Verhältnis Jesu zum AT wahr wie. sonst nirgends. Jede Ausführung würde die Grenzen dieses methodischen Versuchs überschreiten. Es ist auch bekannt, wie weit die grundsätzliche Übereinstimmung gerade bei sonst weit auseinander Gehenden reicht, wenn sie anders die Wirklichkeit des Bildes Jesu ernstlich erfassen und nicht mit fremden, von außen herangebrachten Maßstäben arbeiten; man denke an den kraftvollen Ansatz in Wernle's Jesus über die Bibel Jesu und etwa Schlatters ebendarauf sich beziehende Äußerungen, aber auch schon Kähler's „Jesus und das AT". Hier ist nur darauf hinzuweisen, wie unsre methodische Forderung allen solchen Erörterungen eine starke Grundlage geben würde. Noch sei erwähnt, wie ein absichtliches Eingehen auf das "Wie?" der Wirkung des ATs im NT auch hinsichtlich dieses „Wie“ bedeutsame Unterschiede zwischen der Gemeinde und Jesus innerhalb der weitgehenden Gemeinsamkeit ergeben würde: die Verwertung in Jesu Worten ist die selbständigere, ursprünglichere. Von da aus würde auch für die oben erwähnte Frage sich ein Antwortbeitrag finden, wie weit wir in den doch sämtlich durch die Gemeinde hindurchgegangenen Zeugnissen Jesu nicht von ihr Umgestaltetes zu erkennen vermögen. Ebenso für das gleichfalls schon genannte Problem der Abgrenzung des NTs von der nächstfolgenden christlichen Literatur - das alles im Sinne größerer oder geringerer geschichtlicher Wahrscheinlichkeit verstanden; man denke an die einst von Hofmann entdeckte, von Ritschl aufgenommene, von Harnack für die wichtigsten neutestamentlichen Schriften anerkannte alttestamentliche Bedingtheit. So ist denn am Schlusse dieses Abschnitts die nochmalige Betonung, wenngleich selbstverständlich, doch nicht unnötig, wie die Forderung rein geschichtlicher Untersuchung uns allein in der gemeinten Richtung vorwärts bringen kann. Als Beispiel, wie langsam aber sicher sie fortschreitet, kann ein Blick auf die Psalmenüberschriften in den alten Bibelausgaben für die Gemeinde, in den heutigen, in den Schulbibeln, bei Kautzsch dienen. Wenn es früher bei Psalm 110 hieß: Jesus der ewige König und Hohepriester, heute: der Priesterkönig, so wird man nicht zweifeln, wieviel schon eine so verbesserte Überschrift dem sachgemäßen Verständnis der alttestamentlichen Bedingtheit des NTs dient. 
Lehrreich wäre im Zusammenhang damit eine geschichtliche Darstellung der wichtigsten in der christlichen . Kirche vertretenen möglichen Standpunkte in betreff des Verhältnisses vom AT und NT. Die dogmatistische und die historische Auffassung würde den obersten Einteilungsgrund abgeben. Dabei kann die erstere mit Anerkennung und mit Ablehnung der Offenbarung verbunden sein. Denn dogmatistisch ist die behauptete wesentliche Gleichheit vom AT in der vorreformatorischen Kirche, in der altprotestantischen Orthodoxie, bei Hengstenberg, in manchen freien Gemeinschaften, wie der betonte Gegensatz der Testamente bei Marcion und Schleiermacher, beidemal unter Anerkennung der Offenbarung; dogmatistisch ebenso, unter Ablehnung der Offenbarung, die rationalistische Auffassung unseres Problems. Ein anderer Einteilungsgrund ergäbe sich, je nachdem das AT christianisiert wird oder das NT judaisiert, gleichfalls in mannigfaltiger Weise. Besonders wichtig aber wären die Übergänge zwischen dogmatistischer und geschichtlicher Stellung, und zwar wieder bei allen zuvor genannten Möglichkeiten. Denn vor dogmatistischen Elementen ist auch die grundsätzlich historische Richtung nicht an und für sich gesichert, weder die literarkritische noch die religionsgeschichtliche.

Zum Schluß ist dankbar zu verweisen auf Hühn's „Die messianischen Weissagungen“ (1899 ff.) und Dittmar's „Vetus Testamentum in Novo" (1899 ff.). Wenn das letztere Werk, ausgezeichnet durch genaue Darbietung des Textes, von vornherein über die "messianischen Weissagungen " hinausgreift, so das erste tatsächlich. Wie sich zu diesen unentbehrlichen Arbeiten die hier vorgeschlagene methodisch verhält, ergibt sich aus ihr von selbst.

Nun die zweite Aufgabe: die Bedeutung einer solchen Untersuchung für die einzelnen theologischen Disziplinen. Die unmittelbarste hätte sie selbstverständlich für die Neutestamentliche Wissenschaft. Freilich wird von keiner Seite grundsätzlich bestritten, daß gerade die leitenden neutestamentlichen Anschauungen, wie etwa Reich Gottes, Sohn Gottes, nicht ohne ihre alttestamentlichen Wurzeln richtig aufgefaßt werden können. Aber selbst sie werden keineswegs immer methodisch genau untersucht. Gerade bei der Bestimmung des Gedankens "Sohn Gottes" wird oft in den verschiedenen Lagern auffallend rasch die alttestamentliche Voraussetzung erledigt, bei den einen um ihre dogmatischen, bei den andern um ihre religionsgeschichtlichen Lieblingsgedanken einzutragen, was dann, weil jene ursprünglich von diesen mitbestimmt waren, vielfach auf ein ähnliches 
Ergebnis führt, nur mit der entgegengesetzten Wertung, einer ewig gültigen Glaubenswahrheit oder einer längst überwundenen Zeitvorstellung. Wie wenig genau sogar deutliche Parallelen auch in diesem grundlegenden Punkt, den Glaubensaussagen über Jesus, beachtet werden, mag Mt 28, 18 zeigen: in den Ausgaben des NTs fehlt wohl bis auf die Nestle'sche und in den Kommentaren aller Richtungen Dan 7, 14, obwohl der Text der LXX die entscheidenden Worte enthält, und zwar desto vollständiger, je sorgfältiger man auf den Wechsel in der Übersetzung des aramäischen Textes achtet. Neben dem wichtigen Beispiel sei ein gleichgültiges erwähnt: in Röm 12,9 fehlte bis vor kurzem Ps 97, 10 in Ausgaben wie Erklärungen. Es sei hier aber nur darauf etwas genauer hingewiesen, welche Bedeutung die Erkenntnis der alttestamentlichen Bedingtheit des NTs gerade für die schon eben erwähnte moderne religionsgeschichtliche Exegese hätte. Ihre grundsätzliche Berechtigung steht außer Zweifel, und unsere theologische Bereitschaft, von den Philologen auch hierin dankbar zu lernen, sollte außer Zweifel stehen. Aber es ist kein befriedigender Zustand, wenn, um mit Kleinerem zu beginnen, zu dem "Tisch des Herrn“ 1 Kor 10,21 alle denkbaren, möglichen und unmöglichen Analogien aus dem Religionssynkretismus aufgezählt werden, ohne daß Mal 1, 7.12 auch nur erwähnt wird. Oder wenn das Wort „Fels" überall an Mithrasüberlieferungen erinnern soll, über seinem ganzen reichen alttestamentlichen Gebrauch tiefes Schweigen liegt. Oder, wenn wir zum Wichtigeren gehen, wer wollte die Erkenntnisse unterschätzen, die uns aus den neuesten Untersuchungen über "Sohn Gottes“, „Herr", „Soter“, "Evangelium", „Epiphanie“ und „Parusie“ zugeflossen sind, und zwar wo diese Begriffe sich auf. Größen der Mysterienkulte oder wo sie sich auf den Cäsarenkult beziehen, wenn man sich auch nur daran erinnert, welches Licht dadurch auf eine Stelle wie 1 Kor 8, $6 \mathrm{ff}$. gefallen ist? Ebenso wird jeder Erklärer des Römerbriefs bezeugen, wie viel anschaulicher z. B. Röm 6 geworden ist, seit wir annehmen dürfen, daß die Gedanken vom Sterben und neuen Leben in der damaligen Frömmigkeit weit verbreitet waren. Das Weltevangelium des Weltmissionars wird uns in seiner Anknüpfung wie noch mehr in seinem Gegensatz $\mathrm{zu}$ dem Vorhandenen nach den verschiedensten Seiten in neuer Weise deutlich. Aber die Dankbarkeit für solche Bereicherung unsrer Einsicht in die eine Seite damaliger Wirklichkeit entbindet nicht von der Verpflichtung, sondern verschärft sie, die andre Seite der Wirklichkeit, und dann muß man wohl doch sofort sagen, die ältere, größere und tiefere, genauer die andre Welt der Voraussetzungen aufs 
allergenauste $\mathrm{zu}$ untersuchen. Es erscheint dann nicht einmal als verächtliches Geschäft, zu zählen, wie oft im AT. vom „Retter“ die Rede ist, wieviel von dem "sich offenbaren" Gottes. Oder um noch auf einen andern Kreis von religiösen Grundworten hinzuweisen: die lehrreichen Untersuchungen über "Gnade“, „Licht", „Herrlichkeit" würden vertieft und wohl nicht unwesentlich verändert, wenn die alttestamentlichen Wurzeln bloßgelegt würden. Ein Blick in die LXX-Konkordanz macht es einfach unmöglich, die Stelle Röm 6,7 als Schlüssel des paulinischen Rechtfertigungsgedankens zu erklären. Das ist gerade so unleugbar, als daß durch dieselbe LXX-Konkordanz, insbesondere in bezug auf die Psalmen oder den zweiten"Jesaja, die Auffassung unsrer altprotestantischen Dogmatiker unmöglich wird. Wiewenig aber durch die Losung „zurück ins Alte Testament" eine voreingenommene $\mathrm{Be}$ schränkung auf den Kanon empfohlen werden soll, mag der vorangestellte Gedanke der "Herrschaft Gottes" erläutern. Es wird sich ganz von selbst ergeben, daß die apokalyptische $Z$ wischenliteratur ihren Beitrag zum Verständnis Jesu liefert, wenn man seine. Verkündigung mit dem AT und mit ihr vergleicht. Freilich'wohl noch mehr, wie sie eben beide Größen überbietet, aber im AT ihre stärkeren Wurzeln hat.

Die neutestamentliche Wissenschaft ist selbst Geschichtswissenschaft, Wissenschaft der Anfangsgeschichte unsrer Religion. Das bisher Ausgeführte betraf also die Bedeutung unsrer Disziplin, wenn wir in der Kürze den Einblick in die alttestamentliche Bedingtheit des NTs so heißen wollen, eben für die Geschichtswissenschaft. Es darf aber ausdrückiich angefügt werden, daß ihre Bedeutung auch für die spätere Geschichte des Christentums, die im engeren Sinn sogenannte Kirchengeschichte, sich erweist. Wieder mögen ein paar Beispiele genügen. Aus der Geschichte der christlichen Sittlichkeit das eigenartige Verständnis so wichtiger Gedanken wie Buße, Bekehrung, Wiedergeburt; insbesondere aus der Geschichte der Sozialethik die mannigfaltige Verwendung der Idee Reich Gottes bei Luther, Calvin, Schwärmern, modernen Christlich-Sozialen. Viele Unbestimmtheit, viel bloßes Spiel geistreicher Beziehungen würde zurückgedrängt oder unmöglich gemacht, wenn der Sinn jenes Wortes im NT in seiner. Verwandtschaft mit dem alttestamentlichen und ebendadurch in seiner unterscheidenden Eigenart deutlich erkannt wäre. Nicht kleiner wäre der Gewinn für die Geschichte des Dogma. Man gewönne sicherere Maßstäbe für die „Hellenisierung des Christentums“, wenn erst jene neutestamentlichen Zeugnisse vom "Sohn" in ihrer alttestamentlichen Bodenständigkeit erkannt wären: wieder nicht ohne volle Anerkenntnis auch der andern 
Einflüsse auf ihre Gestaltung. Aufs Ganze gesehen, würde wohl manche Erkenntnis reifen, die von den Lieblingsmeinungen entgegengesetzter Richtungen im Urteil über diese Dinge gleich weit ablägen, aber der geschichtlichen Wirklichkeit näher kämen.

Noch eine allgemeinere Bemerkung mag hier Platz finden, die natürlich auch für die Schriftwissenschaft als geschichtliche gilt. Nämlich daß die vielerörterten und öfter noch ohne genaue Erklärung vorausgesetzten methodischen Grundsätze der Korrelation und Analogie durch ihre Anwendung auf jenem bestimmten Gebiet, der Verwurzelung des NTs im AT, eine genauere Fassung erhielten. Denn die Beobachtung der einigermaßen nachzuprüfenden ursächlichen Zusammenhänge und Verwandtschaften schärft den Blick für das noch Unbekanntere und Fernerliegende und gewährt damit überhaupt erst eine fruchtbare Ausgestaltung jener heuristischen Regeln, sichert ihre wirkliche Bedeutung und bewahrt vor den Erzeugnissen einer zügellosen Phantasie, die überall "Entsprechungen“ sieht, wo keine sind, und „Erklärungen“ des Zusammenhangs, die das Ursprüngliche in der Geschichte verkürzen.

Besonders eingreifend dürfte die Bedeutung der hier empfohlenen Untersuchung für die systematische Theologie sein. Beschränken wir uns auf die dogmatische und auf ihr Grundproblem, die Bindung unsres Glaubens an die Geschichte Jesu. Einst der Entstehungsgrund dieses Glaubens, ist sie für uns zu dem großen Problem geworden, um das sich, oft unausgesprochen, dann nur um so tiefer in innerstem Anteil das Nachdenken der heutigen Theologie bewegt. Davon gingen wir aus. Immer sind im Grunde zwei Fragen in diesem Problem zusammengefaßt. Die eigentlich dogmatische: warum, aus welchen innersten Strukturverhältnissen, tiefsten Bedürfnissen, letzten Motiven des Glaubens ist unser christlicher Gottesglaube nicht von dieser Geschichte lösbar? Warum, wie man so oft, seit die Frage deutlich gestellt wurde, wiederholt hat, ist die Geschichte nicht nur Illustration, sondern Demonstration, Begründung nicht im Sinn des historischen Ursprungs, sondern der inneren Geltung? Und die andere Frage: bietet die Geschichte diese für den Glauben (nach seiner Selbstverständigung über seine Eigenart) unentbehrliche wertvolle Wirklichkeit? Die beiden Fragen hängen enger zusammen, als man sich oft deutlich macht. Nämlich sofern die Gleichgültigkeit oder doch Ungenauigkeit in der Untersuchung der einen Gleichgültigkeit oder doch Ungenauigkeit in der Behandlung der andern nach sich zieht, und diese Sorglosigkeit in bezug auf die eine wieder die in bezug auf die andere steigert, und so fort in immer sich verstärkender Wechselwirkung. Und zwar gilt dies merkwürdig 
unabhängig von den entgegengesetzten theologischen Grundrichtungen. Es ist oft bemerkt worden, daß die Antwort der "orthodoxen" Christologie auf jene erste, im engern Sinn dogmatische Frage den Anteil an jener zweiten, der eigentlich geschichtlichen; geschädigt hat. Man suchte eben nur den Beweis für den feststehenden Glaubenssatz und hatte keinen Blick für das, was damit nicht unmittelbar zusammenhing. Z.B., daß der Jesus der Evangelien Subjekt des Glaubens, der Demut, des Gebets ist, war nur eben ein Zeugnis für das „wahrhaftiger Mensch“, aber ohne liebevolle Versenkung in den anschaulichen Reichtum, ja oft mit starker Verkürzung der neutestamentlichen Aussagen. Und diese unvollständige Benützung des geschichtlichen Stoffs wirkte deutlich zurück auf das Dogma vom Gottmenschen: es wurde immer unlebendiger in sich nach allen Seiten ausgeführt und geschützt, aber ohne Trieb. zur inneren Klärung und Vertiefung; je mehr aber das Bekenntnis zur bloßen Tradition wurde, erlosch wiederum immer mehr das Auge für die geschichtliche Beobảchtung. Es steht jedoch nicht anders mit der "liberalen" Theologie. Überzeugt, daß die Wahrheit des christlichen Glaubens von der 'Geschichte unabhängig ist, weil viel sicherer in dem "religiösen Apriori" verankert, hat auch sie wenig Sinn für die unbefangene Beobachtung des Geschichtlichen. Hingenommen von ihren grundsätzlich so berechtigten Maximen der Korrelation und Analogie, ist sie geneigt, was in der Überlieferung als eigentümlich sich darbietet, auszugleichen; man denke an die weitverbreitete Behandlung des Wortes Jesu vom Bekennen seines Namens. Wie ein solches Verfahren die mit Worten hochgepriesene geschichtliche Unparteilichkeit schädigt, so schwächt es immer mehr die innere Bereitschaft, jene andere Seite des Problems, den etwaigen religiösen Wert einer etwa so besonderen geschichtlichen Größe, immer aufs neue zu erwägen. Und auch hier geht die Wechselwirkung immer fort.

Nun, eben gegenüber diesen Gefahren, die man von jedem Standpunkt aus würdigen kann, dürfte ein Schutzmittel in der rückhaltlosen Untersuchung der alttestamentlichen Bedingtheit unsrer Religion liegen. Ein Schutzmittel, gewiß nicht das einzige, denn weil jenes Problem, wie gezeigt, ein doppelseitiges ist, muß auch seine rein dogmatische Seite immer aufs neue ins Auge gefaßt werden. Es ist erstaunlich, wie wenig in mancher sehr "positiven " Theologie über sie verhandelt wird: als ob es sich von selbst verstünde, warum wir nicht von Jesus loskommen dürfen, auch wenn die in ihrer Art so großartige Antwort der Altẹn keineswegs festgehalten wird. Das gerade ist ja so oft ein Grund der Unfruchtbarkeit auch vieler Predigten, daß sie nicht den 
Eindruck in klarer Verkündigung hervorrufen können: „dich muß ich haben". Dann sind alle hohen wohl- und ernstgemeinten Ehrentitel, die man auf Jesus häuft, umsonst, ja gereichen zum Anstoß. Hier aber haben wir es zunächst mit der andern Seite des Problems zu tun, mit der geschichtlichen Frage nach der Wirklichkeit einer für den Glauben so wertvollen Größe; und selbstverständlich nur mit einem Beitrag zur Lösung dieser Frage. Aber ist nicht wenigstens dies eine auch ohne eingehenden Beweis klar: das in seine alttestamentlichen Wurzeln verfolgte Zeugnis von dem "Sohn" Gottes ist weder mit der "orthodoxen" Deutung dieses Wortes noch mit seiner "liberalen" identisch? Es hat in jenem Zusammenhang einen spezifischen Eigenton und Sondersinn, der, wie alles in sich Eigenartigste, ganz andern Widerstand seiner Auflösung entgegensetzt als jene beiden. Gerade aber in dem so viel genauer umrissenen geschichtlichen Bild läge dann von selbst die Aufforderung, die religiöse Bedeutung einer solchen geschichtlichen Größè neu zu untersuchen, ihren Zusammenhang mit den allereinfachsten, aber ebendarum tiefsten Grundbeziehungen des religiösen Vorgangs selbst aufzudecken. Mit andern Worten, die jüngst von einer theologischen Fakultät gestellte Preisaufgabe: „Inwiefern liegen in der heutigen Erforschung der Geschichte Jesu Motive für eine Neugestaltung der dogmatischen Christologie?" würde an der befürworteten Hilfsdisziplin eine unentbehrliche Voraussetzung ihrer Beantwortung haben.

Damit ist von selbst die Bedeutung einer solchen Hilfswissenschaft auch für die praktische Theologie gegeben. Denn jeder Fortschritt in der Erkenntnis des Wesens unsrer Religion ist ein Fortschritt ihrer praktischen Verwertung, jeder Fortschritt aber in der Erkenntnis ihrer Anfänge (in unsrem Fall durch die Einsicht in ihren Zusammenhang mit dem AT) ist ein Fortschritt in der Erkenntnis ihres Wesens, mag man über diese schwierige Frage nach dem Wesen einer Religion sonst noch so verschieden denken. Bei der Erinnerung an die systematische Theologie ist als Beispiel die Christologie gewählt worden; unwillkürlich gedachten wir dabei schon der praktischen. Denn die Schwierigkeit, die alten christologischen Formeln in der Verkündigung $\mathrm{zu}$ verwerten, ist allgemein anerkannt; so ist zum mindesten das günstige Vorurteil berechtigt, es könnte eine an dem ursprünglichen Sinn des NTs orientierte Predigt neue Wege in den alten ewigen Glauben eröffnen. Und zum Beweis, daß es sich hierbei nicht nur um eine ferne Möglichkeit handelt, mag an Uhlhorns Wort erinnert werden, daß das Zeugnis von Jesus Christus, soweit es überhaupt als 
glaubenerweckend und -stärkend sich bewähre, von der dogmatischen Stellung seiner Verkündiger weithin auffallend unabhängig sei. Sollte das nicht mit eine Folge des schon im stillen wirksamen alttestamentlichen Verständnisses des Neuen sein, zu dessen methodischem Ausbau hier ermuntert wird? Nur auf einen allgemeinen Gewinn, der auf diesen Weg locken dürfte, sei noch hingewiesen: auf die Ermöglichung eines wahrhaftigen Bibellesens der Gemeinde gegenüber dem jetzt oft so zügellos willkürlichen, von halbgebildeten Theologen genährten und mitverschuldeten Schriftgebrauch.

So würde denn das gemeinte Unternehmen ein wenn auch noch so bescheidener Beitrag für eine bessere Zukunft der Theologie und Kirche. Es würde in seinem Teil eine äußerliche Restauration von Vergangenem, nach dem Urteil der gottgelenkten Geschichte für uns nicht mehr Lebensfähigem, ebenso unmöglich machen wie jene vielbesprochene Geheimreligion der Gebildeten oder das tatarme christlichsoziale Gerede oder das müde Warten auf eine kommende Reformation. Die vertiefte Einsicht in das Wesen unsres Glaubens würde nicht mehr erlauben, alles Mögliche Christentum żu heißen, und ebensowenig, unüberbrückbare Trennungsgräben $\mathrm{da} z \mathrm{u}$ ziehen, wo sie nicht vorhanden sind. Die tiefste Bindung und eine noch kaum geahnte Freiheit in unzertrennlicher Einheit würde uns in neuer Weise zuteil, die in den Zeugnissen des NTs uns so mächtig ergreift; dieser ihr unmittelbarer Eindruck würde in seinem Grund und Recht uns bewußt, wenn wir durch Einsicht in ihre alttestamentliche Bedingtheit sie in ihrer Eigenart tiefer erfassen. 